\title{
Optical Properties and Electronic Structure of
}

\section{Copper Zinc Sulfide Nanocrystals}

Arpita Mukherjee", Biswajit Bhattacharyya", Guru Pratheep Rajasekar, Awadhesh Narayan* and Anshu Pandey*

Author Address

Solid State and Structural Chemistry Unit, Indian Institute of Science, Bangalore 560012, India

*Corresponding author

\section{MATERIALS AND METHODS}

Synthetic methods: $\mathrm{Zinc}$ acetate $\left(\mathrm{Zn}\left(\mathrm{CH}_{3} \mathrm{COO}\right)_{2}, 99 \%\right)$, Copper acetate $\left(\mathrm{Cu}\left(\mathrm{CH}_{3} \mathrm{COO}\right)_{2}, 98 \%\right)$, 1-Octadecene (ODE ,technical grade,90\%), Oleylamine (technical grade, 70\%) were purchased from Sigma-Aldrich. All the chemicals were used without any purification.

Preparation of CZS NCs: NCs were prepared in two steps. The first step consisted of the preparation of anion precursor (preparation of sulphur in oleylamine). In Flask 1, Sulphur powder $(0.1 \mathrm{mM}, 3.2 \mathrm{mg})$ was added to a $50 \mathrm{ml}$ three-necked flask containing $3.5 \mathrm{ml}$ oleylamine. This flask was held at $160{ }^{\circ} \mathrm{C}$ under argon for 30 minutes.

In the second step, Zinc acetate $(0.09 \mathrm{mM}, 22.85 \mathrm{mg})$, Copper acetate $(0.01 \mathrm{mM}, 1.81 \mathrm{mg})$ were added to a $50 \mathrm{ml}$ three-necked flask (Flask 2) containing $2 \mathrm{ml} \mathrm{ODE}$ as a solvent and $1 \mathrm{ml}$ oleylamine as a ligand. The reaction mixture was heated to $150^{\circ} \mathrm{C}$ under argon and at this 
temperature, $1.5 \mathrm{ml}$ of the solution in flask 1 was swiftly injected. Following this injection, the NCs were annealed at $100^{\circ} \mathrm{C}$ for $10-15$ minutes. During this annealing period, an additional injection of $1 \mathrm{ml}$ of the contents of flask 1 was made to increase NC size. After the desired size was attained, the reaction mixture was cooled to room temperature.

The same procedure was followed for CZS nanocrystals with other compositions. Composition tuning was achieved by varying the copper acetate and zinc acetate ratio. Typically, a 1:9 $\mathrm{Cu}$ : Zn ratio leads to CZS NCs with a 20\% inclusion of copper while a 5:95 ratio leads to a $10 \%$ copper inclusion. We attribute this to the excessive reactivity of the copper precursor over zinc.

The NCs were cleaned after synthesis (before analysis and characterization) to remove the solvent, excess ligand, and unreacted precursor. The cleaning was done in three steps. Initially, $5 \mathrm{ml}$ of methanol was added to $5 \mathrm{ml} \mathrm{NCs} \mathrm{solutions,} \mathrm{and} \mathrm{the} \mathrm{mixture} \mathrm{was} \mathrm{centrifuged} \mathrm{for} 1$ minute at $1000 \mathrm{RCF}$. The same procedure was repeated two times. In the second step, $5 \mathrm{ml}$ of ethanol and $0.5 \mathrm{ml}$ of methanol were added to the mixture and centrifuged for 1 minute at 1000 RCF. Finally, the supernatant was removed, and an NC precipitate was observed on the walls of the tube. Spectroscopic data were collected by dissolving these cleaned NCs in n-hexane solution (spectroscopic grade).

\section{Cross-section Measurements:}

The absorption cross-section of the material was measured by first dissolving samples in toluene to take absorption spectra. The solvent was then evaporated, and the NC residue was treated with concentrated nitric acid. The resultant solution was diluted to $25 \mathrm{~mL}$ in water. The composition was then determined using inductively coupled plasma spectroscopy (ICP-OES). The particle size distributions were estimated from TEM images. Assuming that, the per unit 
cell volume is constant for zinc sulphide. The extinction coefficient was assumed identical to the optical absorption, as is standard for this size of particles.

\section{XRD Analysis:}

Films for XRD analysis were prepared by dissolving cleaned NCs in Hexane solutions, and then drop-casting on a glass substrate. For further cleaning of the NCs film, a few drops of a mixture of $\mathrm{n}$ - butyl amine and acetone were added. A $0.154 \mathrm{~nm}$ x-ray source was used to collect all the data.

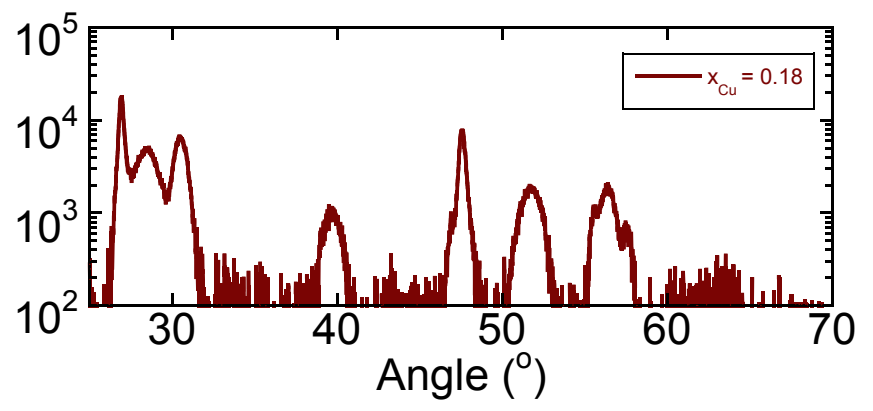

Figure S1: XRD pattern of CZS with $\mathrm{x}_{\mathrm{Cu}}=0.18$ (in logarithmic scale). As evident, parasitic and undesirable phases such as copper sulfides are not detected. Further, given that our noise threshold is at least 2 orders of magnitude below the material signals, we conclude that undesirable phases, if present, constitute less than $1 \%$ of the total ensemble. This is insufficient to perturb or influence our conclusions or produce measurable artifacts within the data presented in this work.
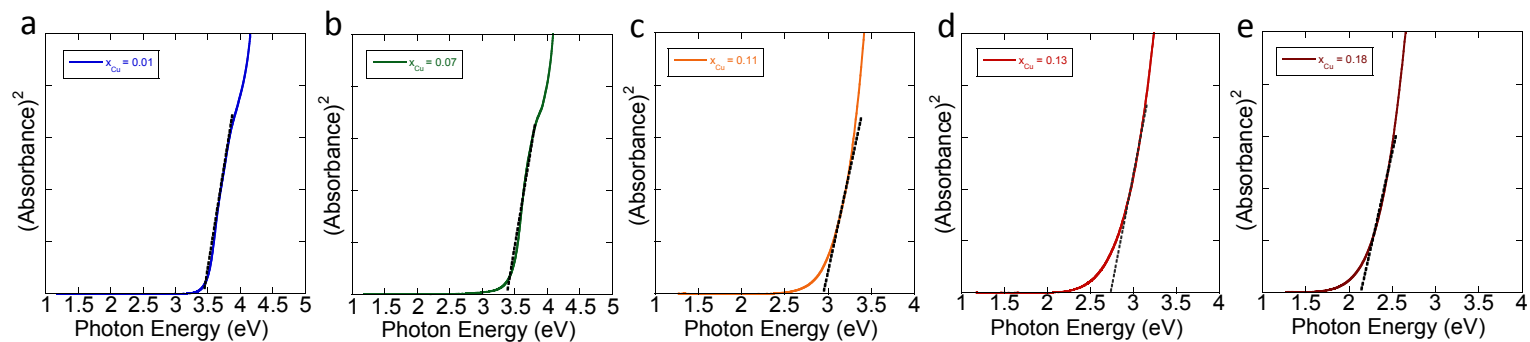
Figure S2 a, b, c, d, e: Tauc plot of different CZS NCs with $\mathrm{x}_{\mathrm{Cu}}=0.01,0.07,0.11,0.13,0.18$, respectively.

\section{Transient Absorption:}

Transient absorption measurements were performed using 100 fs pulses derived from a Coherent Libra amplified femtosecond laser. Samples were stirred to avoid photoinduced charging or degradation effects. Cleaned, purified NCs in a hexane solution were irradiated by a $400 \mathrm{~nm}$ pump obtained by frequency doubling the fundamental. The transient spectra were collected using a broadband white light probe. 